\title{
Sap Data Service for E-Commerce
}

\author{
Pooja Mulam ${ }^{1}$, Ruchi Rautela ${ }^{2}$ \\ ${ }^{1}$ Student VES Institute of Technology \\ ${ }^{2}$ Assistant Professor VES Institute of Technology
}

\begin{abstract}
Now days, everyone is busier with their work life. Therefore a person gets less time to go for shopping. A person gives more preference to online shopping. In the technical world, online shopping refers to E-Commerce. There are many online E-Commerce sites available over web which deal with millions of transactions in a day. These websites deal with huge amount of data which must be maintained for analysing the daily/monthly/yearly transactions, profits and statistics. Data can come from different sources therefore there is a need to refine the data which gives accurate data statistics and provide better report. Here working of data service makes a sense. In this paper we will discuss how SAP data service can be used for E-commerce.
\end{abstract}

Keywords: SAP Data Service, E-Commerce, ETL, Reports

\section{Introduction}

The origin of trade is barter system which includes exchange of goods and services for other goods and services. [1] The invention of money greatly promoted trade by way of exchanging goods and services for money. E-Commerce is buying and selling of products and services by businesses and consumers through an electronic medium, without using any paper documents. There are various ways of electronics payment like electronic wallet, smart card, credit card, debit card, netbanking and more. Now a day, handling a more amount money while travelling is difficult therefore the way of electronics mode of payments becomes useful. Along with this, time matters a lot. Online trading saves a time of a person. This leads to an invention of online business. [2] As many of companies have online presence. In the today's competitive world, conducting online business become necessity for them. There are billions of transactions occurring day to day and companies have to handle these transactions and payments. Also companies have to deal with huge amount of data which are generated through transactions on daily basis to analyse a business. It is complicated to handle this data. Therefore data services are useful to those organisations that uses heterogeneous storage infrastructure using Data as a Service. Data services are software services that encapsulate operations on key data entities of relevance to the enterprise. [3] Data services automate the work of locating heterogeneously-stored data and provide developers and data analysts with simple programmatic tools to find and extract the data they need with little effort. Data services give flexibility for storing data and it is also easy to find and deliver data anywhere. SAP Data Services is a data integration and transformation software application. It allows users to develop and execute workflows that take data from predefined sources called data stores (applications, Web services, flatfiles, databases, etc.) and then allows the user to combine, transform, and refine that data, and then output the results back to the same or different data stores. [4]

\section{Problem Statement}

Every E-Commerce company has goods and services for buying and selling. It has employees, customers, venders along with products. The company needs to maintain information about its employees, customers, venders as well as complete information about goods and services it trades. To maintain this information, database is used by companies. In a database, data is stored in a table with rows and columns. But in E-Commerce websites, data can come from multiple sources. It is a raw data. Sometimes customers do not provide complete information; and the data about venders, employees can be incomplete. For this purpose, the data may require cleansing and additional operations to ensure data quality. Therefore, data warehouse is used as a central repository of integrated data from different sources. It is a most important task for company to manage this data properly. Company needs to maintain current as well as historical data for analysis and creating reports. It is next to impossible to update and maintain huge data manually. Therefore, tool is required to perform these activities.

\subsection{What Is Sap Data Service?}

\section{Proposed Solution}

SAP Data Service is an ETL tool where ETL stands for Extraction, Transformation and Loading of data. ETL is a process of how data extracted from source systems until the data loaded to data warehouse. Fig 1 shows diagramatic representatiom of ETL process. The data is extracted from multiple business applications like 
CRM, Sales, HR and finance and transform a data in staging area. After transformation, data is loaded to central database i.e. data warehouse.

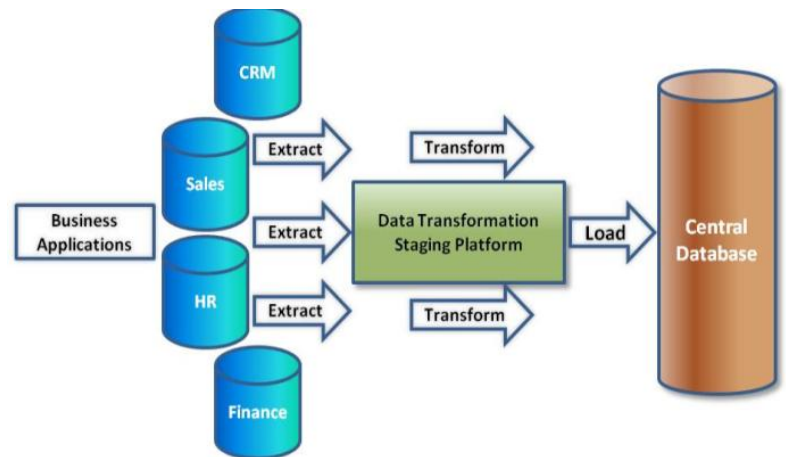

Fig. 1 ETL Process

\subsubsection{Extraction}

During the data extraction stage data is extracted from various source systems and provided to next step. Generally, the data from the source systems is stored 1-on-1 in a 'Staging Area', which will serve as the input for the processing in the transformation phase. [5]

\subsubsection{Transformation}

In a transformation phase, different combinations of functions are used to load data from staging area to data warehouse. Examples of this are the bringing together of similar data from different source systems, the filtering of data, adding new calculations on the basis of data from the source systems, and converting measured values to the same units. [5]

\subsubsection{Loading}

After every transformation is performed, a data is loaded to the end target system which is mostly data warehouse.

\subsection{History}

SAP Data Service is one of the best tools to perform activities required in E-Commerce Websites. SAP Data Services software was originally developed Acta Technology Inc. They developed two different products for Data Integration and Data Quality. In the year 2002, BusinessObject Company acquired Acta Technology Inc. and products rebranded as BusinessObject Data Integration (BODI) and Business Object Data Quality (BODQ). Then in 2007, SAP acquired BusinessObjects and named its products as SAP BODI and SAP BODQ. In 2008, SAP was integrated this two products and named it as SAP BUSINESS OBJECT DATA SERVICES (BODS).

\subsection{Features}

3.3.1 Broad Application and System Support

SAP Data Services provides access to data sources and also provides integration of data sources and targets.

\subsubsection{Processing of native text}

SAP Data Services product has ability to unlock meaning from unstructured text data for increased business insights.

\subsubsection{Intuitive business-user interfaces}

It provides guidance through the process of standardizing, correcting and matching data to reduce duplicates and identify relationships.

\subsubsection{Data quality dashboards}

It displays the impact of data quality problems on all downstream systems or applications.

\subsubsection{Maximized productivity and simplified maintenance}

It provides transformation and cleansing of all types of data, regardless of industry or data domain. It also has ability to utilize a centralized business rule repository and object reuse. 
3.3.6 High performance and scalability

SAP Data Services has ability to meet high volume needs through parallel processing, grid computing and bulk data loading supports. [6]

\subsubsection{Reduces cost}

SAP Data Services boost productivity and cut costs with a single solution for data quality and integration.

\subsubsection{Smart business decisions}

SAP Data Services is useful to make smart business decisions based on trusted information.

\subsubsection{Efficient}

It gains new business process efficiencies with one version of truth across the enterprise.

\subsubsection{Reduce risks}

SAP Data Services reduces risk with consistent and high quality information. [8]

\subsection{Sap Data Services for E-Commerce}

E-Commerce websites involve lots of daily business activities. Data generated by these activities need to be stored somewhere. And the SAP Data Service is best ETL tool to maintain this generated data by websites. The data which is generated and stored at different sources like databases, flat files etc. are extracted and various transformations are performed on data to load it into data warehouse. For this purpose, there is a Job Creation facility available in SAP data service. We can create different jobs for the activities which happened every day. In a job, the name of source file is given and the functions needs to be performed are mentioned, along with this the table name of data warehouse is given to dump a newly generated cleansed data. By creating these daily jobs, the manual efforts of executing the queries, cleansing of data and putting data into tables are reduced. Fig 2 shows job creation functionality. We can write SQL query to extract data from sources. T_IMPERIO_SQL is a table in which data is stored. In a query part, sql query columns and table columns are mapped to each other for proper table format.

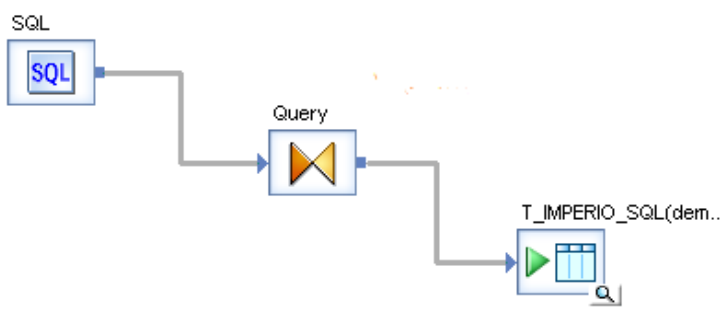

Fig. 2 Job Creation

For an E-Commerce website, some data needs to be generating on regular basis. Therefore the jobs need to be executed regularly. If a company wants to execute a data after specific time interval, then one person in a team has to be alert for executing a job after regular interval. Along with an allocation of one person, the time required to manage a job activity may be more. With the facility of job scheduling in SAP Data Services, this process can be very easy. The jobs can be scheduled for days in a week with specified time interval. Therefore it is very easy to generate a data after specific time interval. Once a job is created and schedule with SAP Data Service, there will be less human interaction with less time. For scheduling a job, SAP provides a separate interface named as SAP Data Service management console. [9] It is a user friendly interface with easy steps to schedule and manage job.(See Fig. 3)

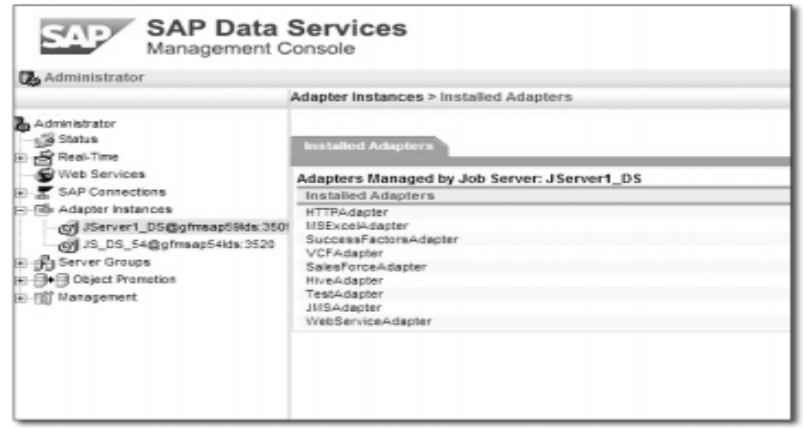

Fig. 3 SAP Data Service Management Console 
The SAP Data Services provides easy and efficient user interface to perform a specific task which involves data manipulation. The objects and functions within BODS are specifically designed to perform manipulations and transformations of huge and complex volume of data very efficiently. There are system provided objects and functions which can be dragged and dropped easily and jobs can be created. This feature of BODS helps Ecommerce companies to make more accurate data manipulation and jobs can be created more efficiently to fulfil the requirements.

E-Commerce websites needs to maintain current as well as past activities of users which help them to track a user, to check their preferences. Tracking a user will helps to provide offers as per user preferences which will help to increase a business of company. For this purpose, companies require to preserve user's data. SAP Data Services has a best option to preserve a data with history preserving feature involves in tool. With the history preserving concept, a data can be store with flag which indicates a current data and historical data. Along with a flag, valid from and valid to columns are also added with data to indicate date ranges within which data is valid. While updating data, data between two tables are compared to decide that the data is newly inserted or it is already existing data. Fig. 4 shows an example of history preserving. The data is extracted from csv or excel file and write a SQL query to move data to table with comparing in table. In a table comparison step, new data from sheet is compared with data in table for maintaining a history. In history preserving step, old data entry flagged as old and new entry as new. Then data in inserted to table with status flag.

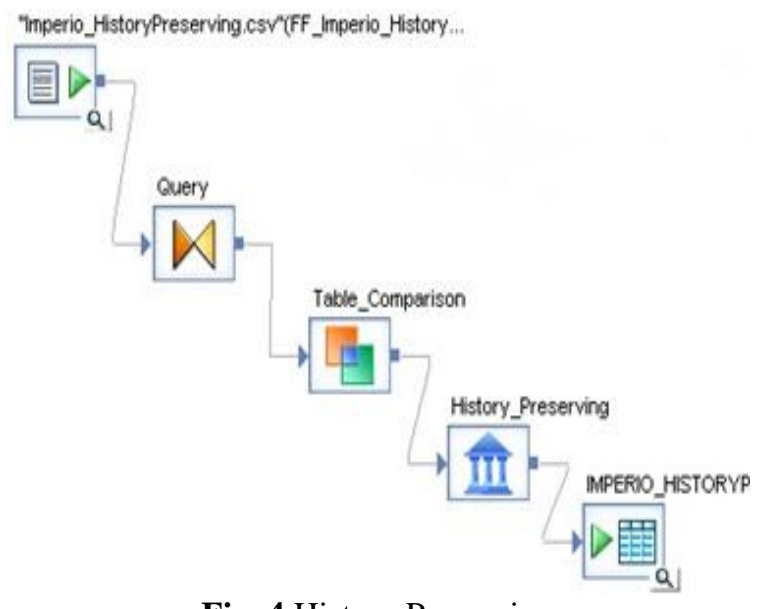

Fig. 4 History Preserving

Since E-Commerce websites deals with many customers, employee and products day to day. It is next to impossible to remember the last customer/employee/product number entered. Also the details of customers are entered online during registration process needs to handle customer number in background. SAP Data Service has a easiest way to handle the auto increment number by using the feature of Key Generation in tool. By using this feature, numbers are generated automatically in incremental manner we have provided. Eventually, it removes the burden of user to remember the number all the time. Fig. 5 shows key generation feature for maintaining data. In a key generation step, unique key is provided for data, which is difficult to remember manually.

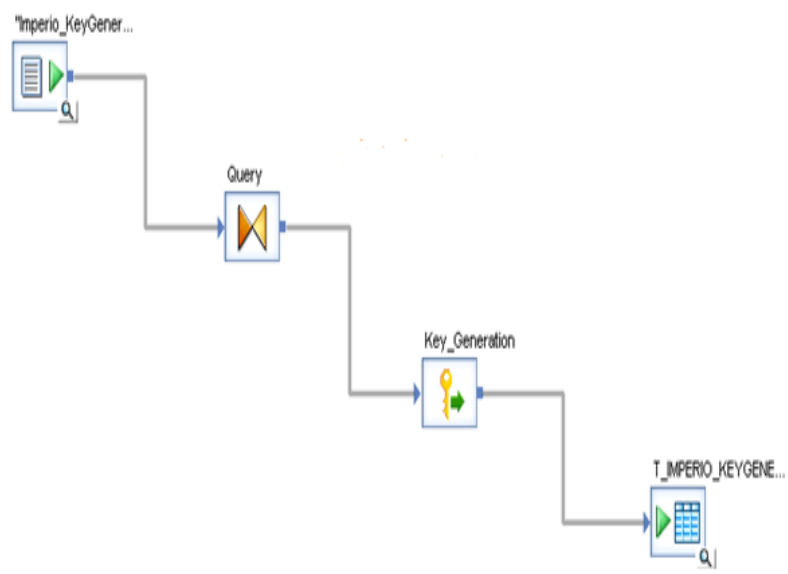

Fig. 5 Key Generation 
SAP Data Service also has features like pivot transform, reverse pivot transform to represent data. Pivot transform is used to convert columns into row format. And Reverse Pivot transform is used to represent row format into column for representing data more precisely. These two transformations are more useful for representing data in table format and report format for better analysis. There are many more features included in SAP Data Service tool which allows organisation to easily explore, extract, transform and deliver data anywhere, SAP Data Service ensure the integrity of your data, maximize developer productivity, and accelerate data integration performance for all operational and analytic initiatives. SAP Data Service is very useful for Business Intelligence activities. It is used for data warehousing and reporting activities which are very easy to analyse the data and comparing performance of company. So that companies can take different approaches to improve the performance and to provide better services.

\section{Conclusion}

SAP Data Service will be very useful tool for E-Commerce companies which help them to manage huge amount of data and also for analysing data. Analysis of data is most important for E-commerce companies to improve the performance and maintain stability in competitive world. With SAP Data Service, Analysis will be easier. Many features are included in SAP Data Service tool which helps developer to maintain data efficiently.

\section{Acknowledgements}

We thank the SAP Data Services developers for initiating such a great product and making it available. We appreciate the efforts performed by them and other people whoever has contributed for making SAP data Services such a big success.

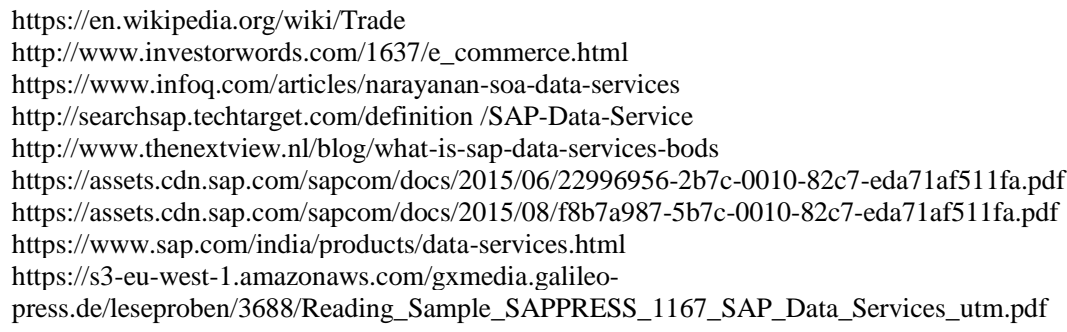

